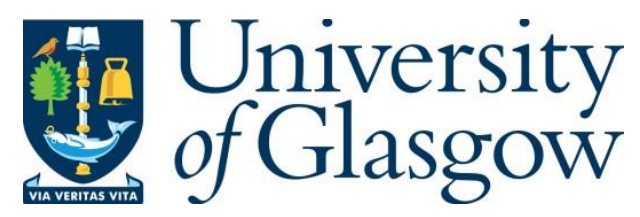

Chen, R. et al. (2018) Variability study of MWCNT local interconnects considering defects and contact resistances - Part II: impact of charge transfer doping. IEEE Transactions on Electron Devices, 65(11), pp. 4963-4970.

There may be differences between this version and the published version. You are advised to consult the publisher's version if you wish to cite from it.

http://eprints.gla.ac.uk/167769/

Deposited on: 28 August 2018

Enlighten - Research publications by members of the University of Glasgow http://eprints.gla.ac.uk 


\title{
Variability Study of MWCNT Local Interconnects Considering Defects and Contact Resistances - Part II: Impact of Charge Transfer Doping
}

\author{
Rongmei Chen, Jie Liang, Jaehyun Lee, Vihar P. Georgiev, Raphael Ramos, Hanako Okuno, \\ Dipankar Kalita, Yuanqing Cheng, Liuyang Zhang, Reetu R. Pandey, Salvatore Amoroso, Campbell Millar, \\ Asen Asenov, Jean Dijon, Aida Todri-Sanial
}

\begin{abstract}
In this paper, the impact of charge transfer doping on the variability of MWCNT local interconnects is studied by experiments and simulations. We calculate the number of conducting channels of both metallic and semiconducting CNTs as a function of Fermi level shift due to doping based on the calculation of transmission coefficients. By using the MWCNT compact model proposed in Part $I$ of this paper, we study the charge transfer doping of MWCNTs employing Fermi level shift to reduce the performance variability due to changes in diameter, chirality, defects and contact resistance. Simulation results show that charge transfer doping can significantly improve MWCNT interconnect performance and variability by increasing the number of conducting channels of shells and degenerating semiconducting shells to metallic shells. As a case study on a MWCNT of $11 \mathrm{~nm}$ outer diameter, when the Fermi level shifts to $0.1 \mathrm{eV}$, up to $\sim 80 \%$ of performance and standard deviation improvements are observed. Furthermore, a good match between experimental data and simulation results is observed, demonstrating the effectiveness of doping, the validity of the MWCNT compact model and proposed simulation methodology.
\end{abstract}

Index Terms-multi-walled carbon nanotubes, charge transfer doping, defects, Fermi level, variability, Monte Carlo simulation.

\section{INTRODUCTION}

A $\mathrm{S}$ an alternative back-end-of-line material, carbon nanotubes (CNTs) present a viable opportunity due to their ballistic transport, high thermal conductivity, and ampacity [1], [2]. Compared to copper lines, CNTs are more resilient and immune to electromigration (EM) as they can sustain significantly larger current densities than $\mathrm{Cu}$ [3]. Furthermore, the resistance of CNT interconnects can be reduced by applying doping such as charge transfer internal or external doping. Doping improves CNT conductivity through the Fermi level,

This work was supported by the European Commission H2020 CONNECT project under grant agreement number 688612. (http://www.connecth2020.eu/).

R. Chen, J. Liang, R. R. Pandey and A. Todri-Sanial are with the Microelectronics Department, LIRMM, University of Montpellier, CNRS, Montpellier, 34095 France. E-mails: rongmei.chen@lirmm.fr; aida.todri@lirmm.fr.

R. Ramos and J. Dijon are with the University Grenoble Alpes/CEALITEN, Grenoble, France.

J. Lee, V. P. Georgiev, and A. Asenov are with the School of Engineering, University of Glasgow, Glasgow, G12 8QQ UK

H. Okuno and D. Kalita are with the University Grenoble Alpes/CEAINAC, Grenoble, France.

S. Amoroso and C. Millar are with Synopsys, Ltd., Glasgow, UK.

Y. Cheng and L. Zhang are with the School of Electronic and Information Engineering, Beihang University, Beijing, China, 100191.

Manuscript submitted May 2018.
$E_{f}$ shift to increase the density of charge carriers (by either holes for p-type doping or electrons for n-type doping) [4][7]. It was also experimentally demonstrated that doping could improve CNT conductivity regardless of CNT diameter and type (either metallic or semiconducting) as in [4]. In [8], it was shown that the addition of MoOx, which forms a composite material with CNT, makes the improved conductivity of doped CNT stable in air at the temperatures up to $390{ }^{\circ} \mathrm{C}$. Similar stable doping has also been achieved by utilizing a single electron oxidant to efficiently dope CNT films by [9].

The shift of $E_{f}$ of CNT can be controlled or tuned by chemical (alkali metals, acids, halogens, ...) [10] or electrochemical doping [11]. In theory, the $E_{f}$ of semiconducting $\mathrm{CNT}$ is at the equipotential position between the conduction and valence band, obtaining p-type and n-type doping with electron-borrowing and donating effects, respectively. Generally, the doping procedure involves the immersion of CNTs into a concentrated solution of dopant for a period of time or exposure to an atmosphere for gaseous dopants [7]. Depending on either the electronegativity for atomic dopants or the electrochemical potential for molecules and inorganic materials, por n-type dopants can be achieved. Due to reactions with the oxygen in the air under ambient conditions, CNTs are slightly p-doped. Therefore p-type doping of CNTs is more efficient and practical to improve CNT electrical properties. There are many kinds of p-type dopants, including $\mathrm{NO}_{2}$ [12], $\mathrm{H}_{2} \mathrm{SO}_{4}$ and $\mathrm{SOCl}_{2}$ [13], and the combination of $\mathrm{HNO}_{3}$ and $\mathrm{SOCl}_{2}$ [14], etc. [7]. Recently, iodine [15], $\mathrm{MoO}_{3}$ [16] and $\mathrm{PtCl}_{4}$ [17] based p-type charge transfer doping have been presented. Charge transfer doping is based on Van der Waals interaction. Compared to substitutional doping, where the covalent bonding between carbon and external atoms forms a bamboolike morphology [18], the charge transfer doping does not modify the CNT structure and hence reduces morphological defects and avoids unexpected physical property changes due to structural modifications. In this work, we study $\mathrm{PtCl}_{4}$ charge based doping.

However, the back-end-of-line processing of CNTs is not yet streamlined or CMOS compatible. CNTs require much higher temperature compared to $\mathrm{Cu}$ (up to $900{ }^{\circ} \mathrm{C}$ ) [19] to achieve good quality CNT growth. Nevertheless, to achieve CMOS BEOL process compatibility, CNTs need to grow at lower temperatures such as $450{ }^{\circ} \mathrm{C}$ to $500{ }^{\circ} \mathrm{C}$. However, at such temperatures CNTs have many defects, variations 
in diameter and chirality, which currently limit the practical application of CNTs as BEOL interconnects. Contacts are also a serious challenge for both reliability (good contact) and performance (high resistance). Metal electrodes might not be connected to all MWCNT shells, which detriments MWCNT interconnect performance.

While there is an extensive body of work on various methods for doping CNTs, there is not yet a systematic study of doping impact on MWCNT interconnects and their variability. Doping is capable of changing semiconducting CNTs into metallic CNTs concerning their electrical performance, and thus it is possible to reduce chirality variation and overall MWCNT resistance variation. In [20], we showed by atomistic-level simulation and experimental results how doping alters CNT chirality. However, there is still a need for further investigations to understand the impact of doping in the presence of various sources of variations such as defects, diameter, chirality, and connectivity.

In this work, we evaluate MWCNT interconnects performance and variability while considering the impact of doping. We introduce the parameter number of conducting channels $\left(N_{C}\right)$ and incorporate it into our enhanced MWCNT compact model described in Part I. By theoretically calculating $N_{C}$ as a function of Fermi level shift $E_{f}$ - we evaluate each source of variation (diameter, chirality, defects) and all-sources using Monte Carlo (MC) simulations. Simulation results are subsequently compared with experimental results, and a good consistency is found between them. The contributions of our work can be summarized as follows.

1) We propose an analytical method for calculating $N_{C}$ for metallic and semiconducting CNT shells and incorporate it into the compact model of MWCNT.

2) We perform MC simulations to study the impact of doping with different variations such as diameter, chirality, defects (defect density) and contacts. Finally, we assess the effectiveness of doping to mitigate the effects of process variations.

3) We present experimental results of MWCNT resistance before and after doping and compare them with simulation results. Good consistency is found between them, demonstrating the correctness of the simulation methodology and the proposed MWCNT compact model.

\section{Analytical Method for Computing the Impact OF DOPING}

Here, we present the analytical method for computing the impact of doping on $N_{C}$ via the Fermi level shift $E_{f}$. The band structure of a zigzag CNT with chirality of $(m, 0)$ can be described by Equation (1) [21]:

$$
E\left(k_{x}\right)= \pm \frac{3 t a_{0}}{2} \sqrt{k_{x}^{2}+\left(\frac{1}{D_{C N T}}\left(2 v-\frac{4}{3} m\right)\right)^{2}}
$$

where $D_{C N T}$ is the CNT diameter, $k_{x}$ is the wave vector in the $x$ direction, $t$ is the hopping parameter, $a_{0}$ is the carboncarbon distance, and $v$ is an integer less than $m . E_{f}$ is assumed to be $0.0 \mathrm{eV}$ for pristine zigzag $\mathrm{CNT}$ although manufactured

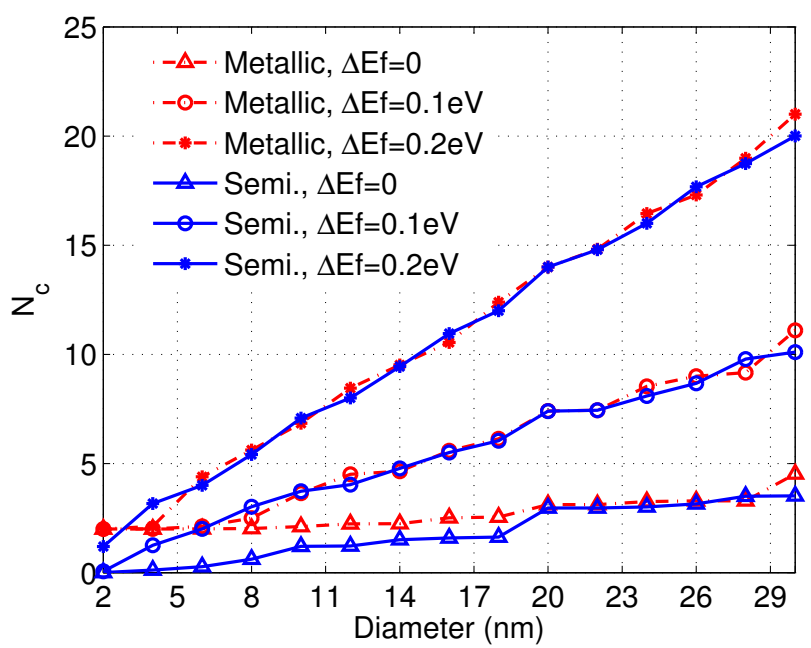

Fig. 1. Change of $N_{C}$ with diameter for metallic and semiconducting CNTs before and after the $E_{f}$ shift.

CNTs tend to be slightly p-doped by the oxygen in the air under ambient conditions [22] and have some downshift of $E_{f}$. From Equation (1), we can calculate the transmission coefficients $(T(E))$, and then $N_{C}$ can be obtained as in Equation (2) [21]. It should be noted that the $N_{C}$ of metallic armchair CNT can be calculated similarly [23].

$$
N_{C}=\int T(E) f^{\prime}\left(E, E_{f}\right) d E /\left(k_{B} T\right)
$$

where $f^{\prime}$ is the derivative of the Fermi function, $k_{B}$ is the Boltzmann constant, and $T$ is the temperature. $N_{C i}$ of the CNT shell $i$ in MWCNT modifies its intrinsic resistance $R_{i}$ as Equations (3) and (4) [24] where $h /\left(2 e^{2}\right) \sim 12.9 \mathrm{k} \Omega$ and $R_{S i}, R_{Q}$ and $\lambda_{i}$ represent scattering and quantum resistances, and mean free path (MFP) of the CNT shell $i$, respectively. $L$ is the MWCNT length.

$$
\begin{gathered}
R_{i}=R_{Q}+R_{S i} L=\frac{h}{2 e^{2} N_{C i}}+\frac{h}{2 e^{2} N_{C i}} \frac{L}{\lambda_{i}} \\
\lambda_{i} \approx 1000 D_{C N T}
\end{gathered}
$$

Calculated results for metallic and semiconducting CNTs of various diameters before and after the $E_{f}$ shift are shown in Fig. 1. It shows that the increase in $N_{C}$ is more significant for larger diameter CNTs than for smaller diameter CNTs. Moreover, metallic and semiconducting CNTs have almost the same $N_{C}$ when their diameters are larger than $20 \mathrm{~nm}$. The increase in $N_{C}$ leads to increase in both conductance and quantum capacitance of MWCNT [24] and can change the performance of MWCNT interconnect.

In Fig. 2 we show the calculated results for different shells of an MWCNT with $11 \mathrm{~nm}$ outermost diameter. The negative shift of $E_{f}$ corresponds to the p-type doping while the positive shift corresponds to the n-type doping. There are in total nine shells based on the shell number calculation equations (in Part I). The smallest or innermost diameter is $5.56 \mathrm{~nm}$. Furthermore, we note from Fig. 2(c) that when $E_{f}$ is shifted 


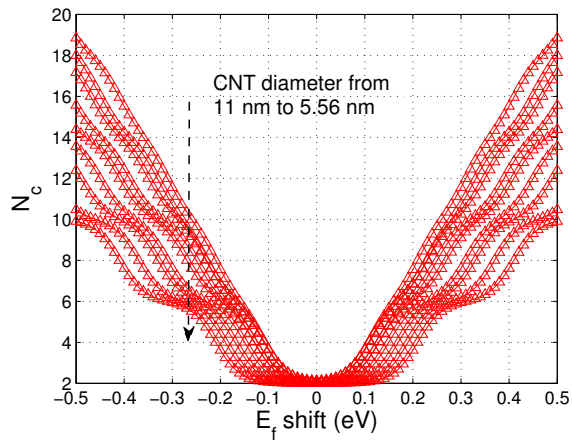

(a) Metallic shells for $D_{\max }=11 \mathrm{~nm}$ MWCNT.

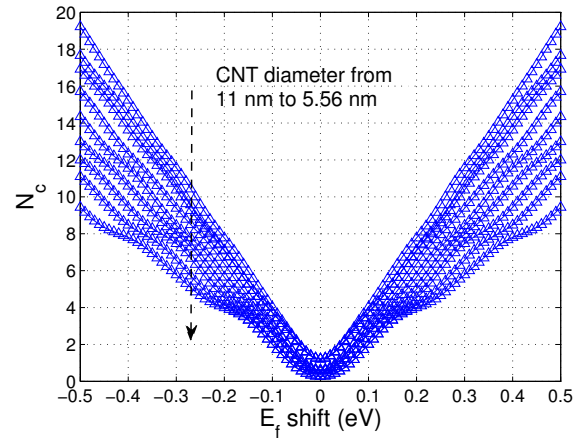

(b) Semiconducting shells for $D_{\max }=11 \mathrm{~nm}$ MWCNT.

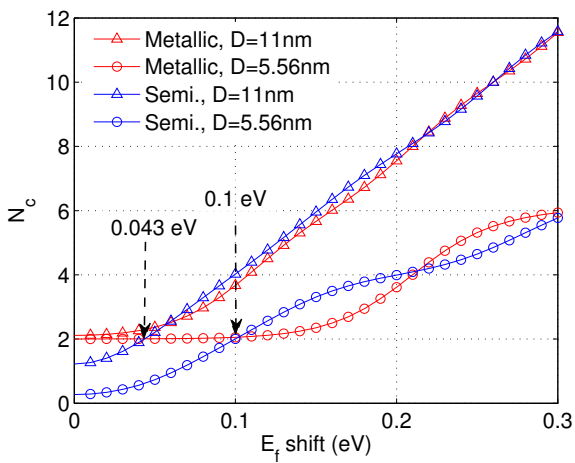

(c) $D_{C N T}=11 \mathrm{~nm}$ and $5.56 \mathrm{~nm}$ shells.

Fig. 2. $N_{C}$ of a) metallic shells with the $E_{f}$ shift, b) $N_{C}$ of semiconducting shells with the $E_{f}$ shift for the MWCNT with $11 \mathrm{~nm}$ outermost diameter, and c) $N_{C}$ of metallic and semiconducting shells of the outermost $(11 \mathrm{~nm})$ and the innermost $(5.56 \mathrm{~nm})$ shells before and after the $E_{f}$ shift $($ similar for the negative $E_{f}$ shift).

TABLE I

REPRESENTATIVE $E_{f}$ SHIFTS FOR MWCNT WITH $D_{\max }=11$ NM AND THE $N_{C}$ FOR METALLIC $\left(N_{C m}\right)$ AND SEMiCONDUCTING $\left(N_{C s}\right)$ CNT SHELLS. ALSO SHOWN ARE THE RATIOS OF METALLIC CNT SHELLS AT DIFFERENT $E_{f}$ SHIFTS.

\begin{tabular}{cccccc}
\hline$E_{f}$ shifts $(\mathrm{eV})$ & 0 & 0.043 & 0.1 & 0.2 & 0.3 \\
\hline $11 \mathrm{~nm} N_{C s}$ & 1.2 & 2.0 & 4.0 & 7.8 & 11.6 \\
\hline $11 \mathrm{~nm} N_{C m}$ & 2.1 & 2.3 & 3.7 & 7.6 & 11.5 \\
\hline $5.56 \mathrm{~nm} N_{C s}$ & 0.27 & 0.61 & 2.0 & 4.0 & 5.8 \\
\hline $5.56 \mathrm{~nm} N_{C m}$ & 2.0 & 2.0 & 2.1 & 3.6 & 5.9 \\
\hline Metallic shell ratio after doping & $1 / 3$ & $1 / 3$ & 1 & 1 & 1 \\
\hline
\end{tabular}

to $0.043 \mathrm{eV}$ and $0.1 \mathrm{eV}$, the outermost and the innermost semiconducting CNT shells start to have $N_{C}=2$, respectively.

Interestingly, pristine metallic CNTs also have $N_{C}=2$ when their diameters are small $(\leq 11 \mathrm{~nm}$, see Fig. 1). Hence, we deduce that a semiconducting CNT of a small diameter $(\leq$ $11 \mathrm{~nm}$ ) becomes equivalent to a pristine metallic CNT when its $N_{C}$ reaches $2\left(N_{C}=2\right)$ by shifting $E_{f}$. Additionally, we assume that the $E_{f}$ shift is the same for both metallic and semiconducting CNT shells because no hint of doping selectivity for semiconducting and metallic CNTs is observed [4]. Furthermore, doping is assumed to have no impact on defect and contact resistance. Based on these assumptions and the simulations shown in Fig. 2(c), one can predict that a MWCNT with $11 \mathrm{~nm}$ outer diameter starts to degenerate semiconducting shells and increase metallic behavior when $E_{f}$ is shifted by $0.043 \mathrm{eV}$ with doping. All shells start behaving as metallic when $E_{f}$ is shifted by $0.1 \mathrm{eV}$.

It is important to note that chirality which represents the carbon nanotube rotation angle does not change with doping - it is the $N_{C}$ and electrical conductance of semiconducting shells that changes to metallic-like shell properties. Detailed information of $N_{C}$ and the ratios of metallic CNT shells in the MWCNT at some specific $E_{f}$ shifts are shown in Table I.

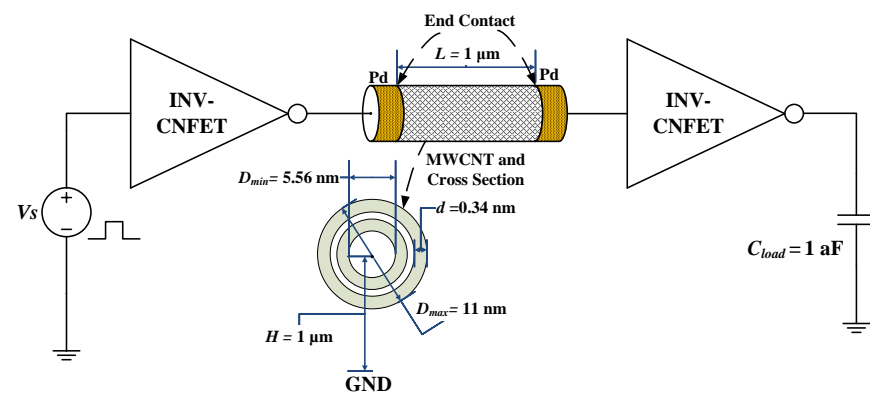

Fig. 3. Simulation setup schematic of MWCNT as interconnect. MWCNT is connected between two inverters composed of CNTFETs.

\section{Simulation Setup And Results}

\section{A. Simulation Setup}

The simulation setup schematic is shown in Fig. 3, which is the same as in Part I of this paper. The circuit benchmark consists of two inverters connected through either an ideal wire or a MWCNT interconnect. Inverter gates are created with carbon nanotube field effect transistors (CNTFETs) based on compact models as in [25]. The key parameters of both n-type and p-type CNTFET are $11.7 \mathrm{~nm}, 1 \mu \mathrm{m}, 10 \mathrm{~nm}$ for gate length, gate width and the distance between CNTs, respectively where other parameters are the default values recommended by [25]. We compute the MWCNT interconnect delay, power consumption, and power-delay product (PDP).

We conduct MC simulations for diameter, defects, chirality and all-sources variations. A detailed description of each variation source can be found in Part I of this paper. One thousand samples are collected to obtain a good confidence level for each simulation condition. We investigate MWCNT doping by considering the five representative $E_{f}$ shifts as listed in Table I. For any given Fermi shift $E_{f}$ (or doping concentration), the MWCNT compact model (described in Part I) is updated to take into account the $N_{C}$ of each shell (either metallic or semiconducting). We also assume a defect density on each shell as $10 / \mu \mathrm{m}$. 


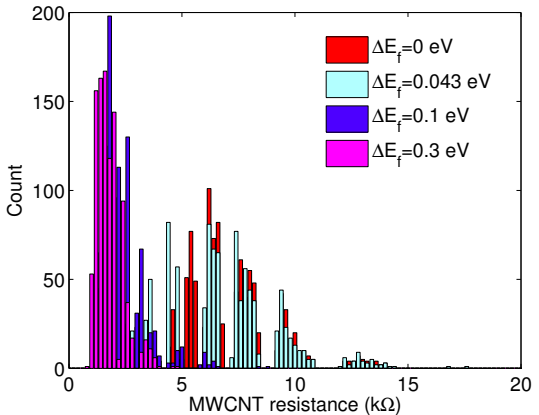

(a) Diameter

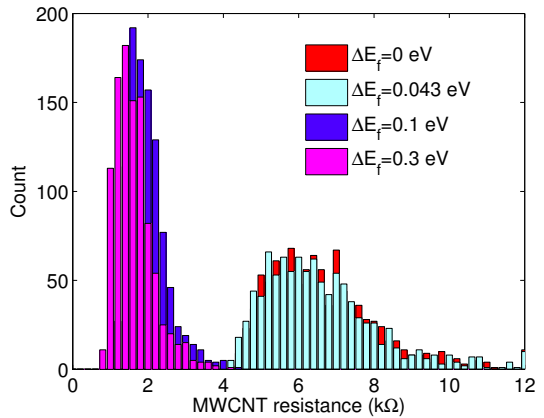

(b) Defects

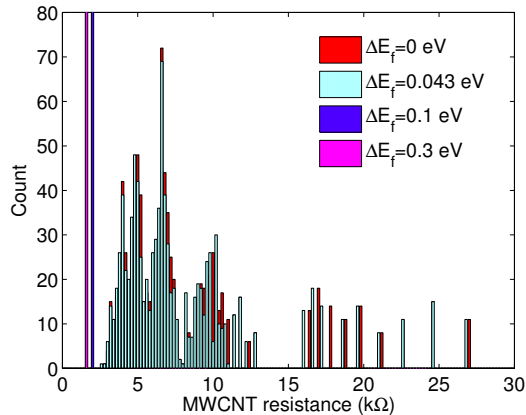

(c) Chirality

Fig. 4. (a)-(c) are distributions of resistance variation of the MWCNT interconnect at different $E_{f}$ shifts with input diameter, defects, and chirality variations respectively.

\section{B. Impact of MWCNT Doping}

In this work, we explore charge transfer doping as a means for reducing resistance variability. As described in Section II, doping shifts the $E_{f}$ and introduces additional $N_{C}$ for both metallic and semiconducting MWCNT shells (see Table I). We perform MC simulations for each source of variation (diameter, defects, and chirality) with different levels of $E_{f}$ for doping. Results are shown in Fig. 4(a)-(c). The all-sources variation results are shown in Fig. 5. Foremost, we observe that doping lowers MWCNT resistance and narrows the distributions, particularly for the $E_{f}$ shift from $0.043 \mathrm{eV}$ to 0.1 eV. Similar trends are also obtained for MWCNT performance (delay, power, and PDP) and are not shown here.

We also calculated the mean value $(\mu)$, standard deviation $(\sigma)$ and $3 \sigma$ percentage $(3 \sigma / \mu \times 100 \%$, used to estimate the largest possible percentage deviation from $\mu$ [26], [27]) of MWCNT resistance and performances with the $E_{f}$ shift and are presented in Fig. 6 and Fig. 7(a)-(c), respectively for each source of variations. Based on our analytical method

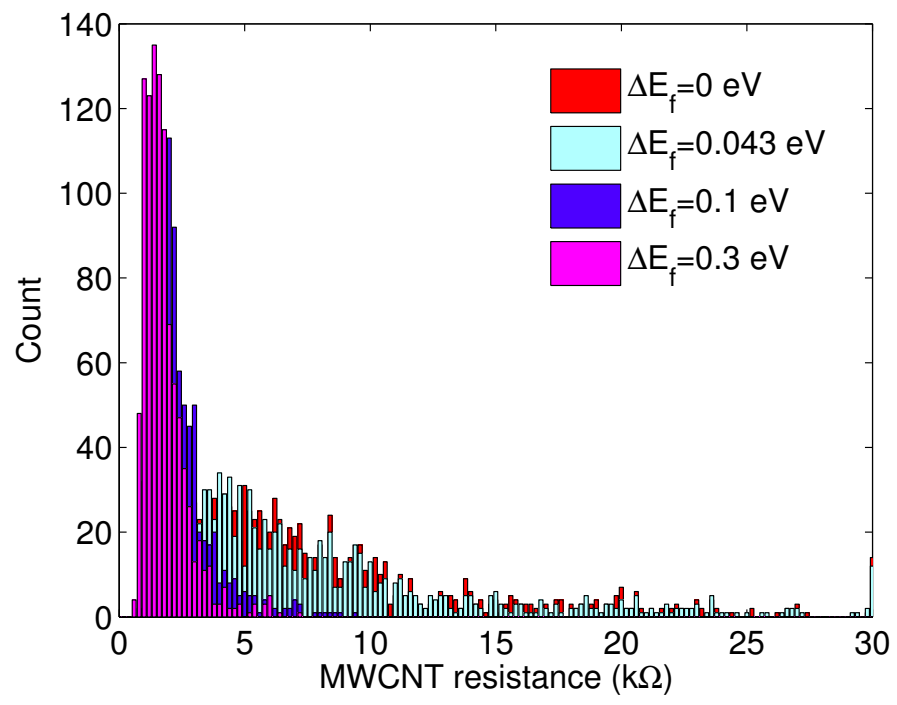

Fig. 5. Distributions of resistance variation of the MWCNT interconnect at different $E_{f}$ shifts with input all-sources variation. from Section II (Fig. 2(c)), we know that doping of $E_{f} \geq$ $0.043 \mathrm{eV}$ starts to increase $N_{C}$ for metallic CNTs, whereas semiconducting CNTs begin to behave as metallic-like shells as $N_{C} \geq 2$. Hence, it is not until $E_{f}$ reaches $0.043 \mathrm{eV}$ that we start to observe improvements in MWCNT resistance and performances $\mu$ and $\sigma$ as in Fig. 6. On the contrary, the $\sigma$ and $3 \sigma$ percentage of diameter variation increases to some extent when the $E_{f}$ shift increases beyond $0.043 \mathrm{eV}$. This is because the outermost semiconducting shell of MWCNT at these $E_{f}$ shifts can behave like metallic CNT shell when its diameter is larger than $11 \mathrm{~nm}$ as a result of diameter variation (see Table I and Fig. 1). The co-existence of metallic and semiconducting CNT shells makes the diameter variation more predominant. Due to the increase in diameter variation, the allsources variation also slightly increases with $E_{f}$ beyond 0.043 $\mathrm{eV}$.

But, as $E_{f}$ reaches $0.1 \mathrm{eV}$, all semiconducting shells behave as metallic, thus improving significantly MWCNT resistance $\mu$ and $\sigma$. As more doping is applied with $E_{f}$ from $0.1 \mathrm{eV}$ to

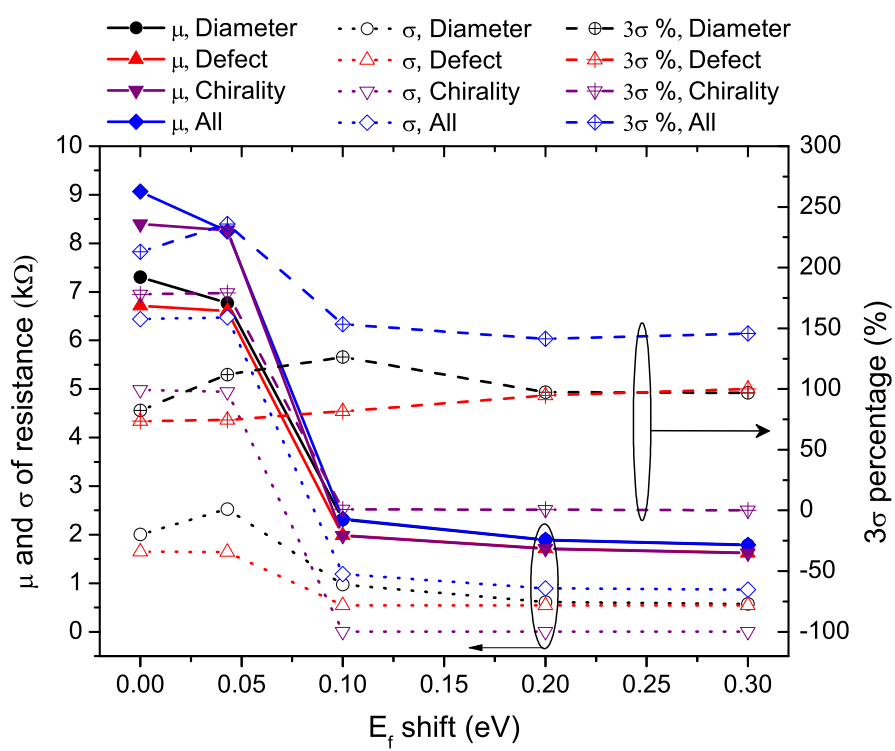

Fig. 6. Change of resistance of the MWCNT interconnect with the $E_{f}$ shift for diameter, defect, chirality and all-sources variations. 


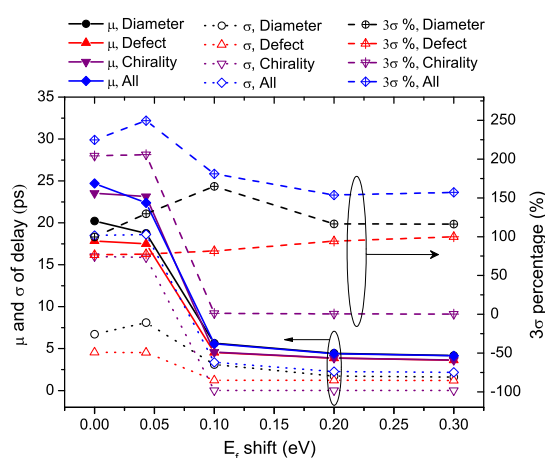

(a) Delay

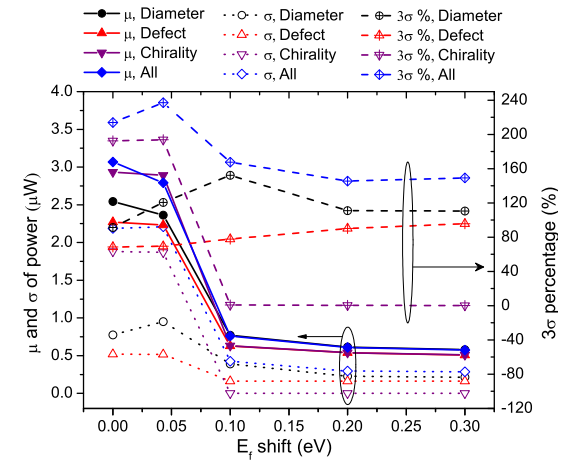

(b) Power

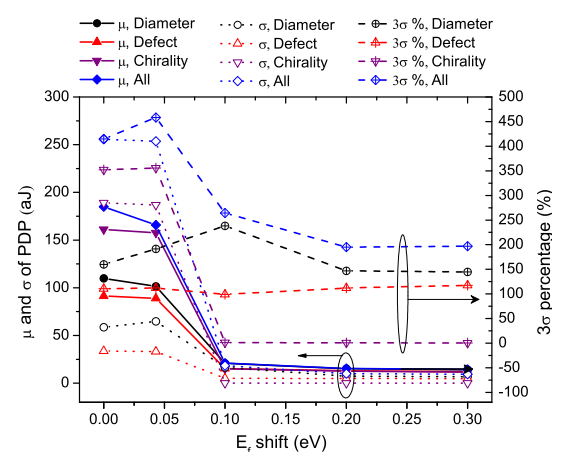

(c) PDP

Fig. 7. (a)-(c) are change of delay, power and PDP variations of the MWCNT interconnect, respectively with the $E_{f}$ shift for diameter, defect, chirality and all-sources variations.

$0.3 \mathrm{eV}$, slight reductions in $\mu$ and $\sigma$ are observed. This can be mainly explained due to two doping attributes: (1) significant CNT shell resistance reduction is obtained at $E_{f}=0.1 \mathrm{eV}$ as all semiconducting shells behave as metallic shells, and (2) with more doping $E_{f} \geq 0.1 \mathrm{eV}$ the $N_{C}$ increases, however, resistance reductions is less drastic as in (1). Additionally, the change in the ratio of metallic to semiconducting CNT shells makes the chirality variation reduced (both $\sigma$ and $3 \sigma$ percentage). It approaches zero when $E_{f}$ reaches $0.1 \mathrm{eV}$. We also note that although the $\sigma$ of diameter and defects variation reduce with $E_{f}$ of $0.1 \mathrm{eV}$, the $3 \sigma$ percentage does not improve due to their similarly decreasing rate of $\mu$. Both diameter and defect variations remain present as $E_{f}$ shifts beyond $0.1 \mathrm{eV}$ because they are not directly related to $E_{f}$ or $N_{C}$ and furthermore contact and defect resistances (contribute to diameter and defects variations) are assumed to be not impacted by doping in this work. Hence, their impact cannot be canceled out by doping.

All-sources variation shows a similar trend in resistance and performances $\sigma$ to that of chirality variation with the $E_{f}$ up to $0.1 \mathrm{eV}$ as shown in Fig. 6 and 7. Beyond $E_{f}$ of $0.1 \mathrm{eV}$, the contribution of chirality to overall variation is negligible, but the variations of diameter and defects dominate. We can deduce that doping helps to reduce overall MWCNT variation by decreasing the contribution of chirality variation mainly due to the degeneration of semiconducting shell properties to metallic. Doping has limited impact on defects and diameter (including contact resistance variation) variations, especially for the $3 \sigma$ percentage variation.

\section{Comparisons with Experimental Data}

As described in [20], we developed a CNT integration process to grow individual MWCNTs at predefined locations on a silicon wafer by hot filament assisted CVD, and to individually contact them with Palladium (Pd) electrodes for electrical characterization. External doping of the MWCNT by $\mathrm{PtCl}_{4}$ salt was then developed. To estimate the impact of doping under various conditions, similarly to [20], we assume the contact resistance is much smaller than MWCNT intrinsic resistance and compare the distributions of linear resistance before and after doping. In [20], a 40\% reduction

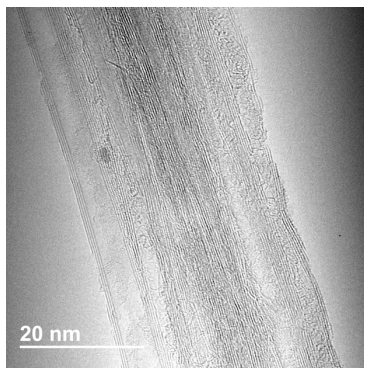

(a) Before doping

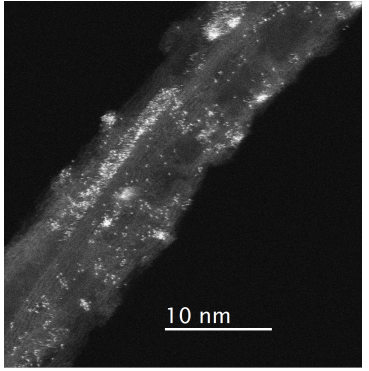

(b) After doping
Fig. 8. TEM and STEM pictures for MWCNT before and after doping with $\mathrm{PtCl}_{4}$, respectively.

TABLE II

COMPARISONS OF DOPING EFFICIENCY IN REDUCING MWCNT RESISTANCE BETWEEN THE ALL-SOURCES SIMULATION RESULTS AND THE EXPERIMENTAL DATA IN THIS WORK OR THAT FROM [20].

\begin{tabular}{ccccc}
\hline $\begin{array}{c}\text { Doping } \\
\text { process }\end{array}$ & $\begin{array}{l}\text { Non } \\
\text { doped }\end{array}$ & $\begin{array}{l}\text { Dipping } \\
{[20]}\end{array}$ & $\begin{array}{l}\text { Spray } \\
\text { dopant 1 }\end{array}$ & $\begin{array}{l}\text { Spray } \\
\text { dopant 2 }\end{array}$ \\
\hline Estimated $E_{f}$ shifts $(\mathrm{eV})$ & 0.00 & 0.104 & 0.122 & 0.250 \\
\hline$\sigma$, simulation & $0 \%$ & $-19.6 \%$ & $-42.6 \%$ & $-77.2 \%$ \\
\hline$\sigma$, experiments & $0 \%$ & $-18.6 \%$ & $-59.3 \%$ & $-77.2 \%$ \\
\hline$\mu$, simulation & $0 \%$ & $-41.2 \%$ & $-55.1 \%$ & $-73.1 \%$ \\
\hline$\mu$, experiments & $0 \%$ & $-41.4 \%$ & $-55.5 \%$ & $-73.3 \%$ \\
\hline $3 \sigma \%$, simulation & $0 \%$ & $38.9 \%$ & $24.4 \%$ & $-16.6 \%$ \\
\hline $3 \sigma \%$, experiments & $0 \%$ & $39.0 \%$ & $-8.5 \%$ & $-14.5 \%$ \\
\hline
\end{tabular}

of the linear resistance was obtained by dipping the connected MWCNTs in $\mathrm{PtCl}_{4}$ solution. In this work, we used a different doping process and instead sprayed the $\mathrm{PtCl}_{4}$ solution on the connected MWCNTs to better control the amount of dopant on the CNTs. It is found that both mean value and variations of MWCNT resistance are significantly reduced after doping. In addition, higher doping efficiencies can be obtained with the spray doping process and that the Fermi level shift can be controlled by the amount of dopant deposited on the CNTs. TEM and STEM pictures of MWCNT before and after doping are shown in Fig. 8.

The measured average $D_{\max }$ of the experimentally doped MWCNTs was $6.5 \mathrm{~nm}$. Because the defect density and MFP 


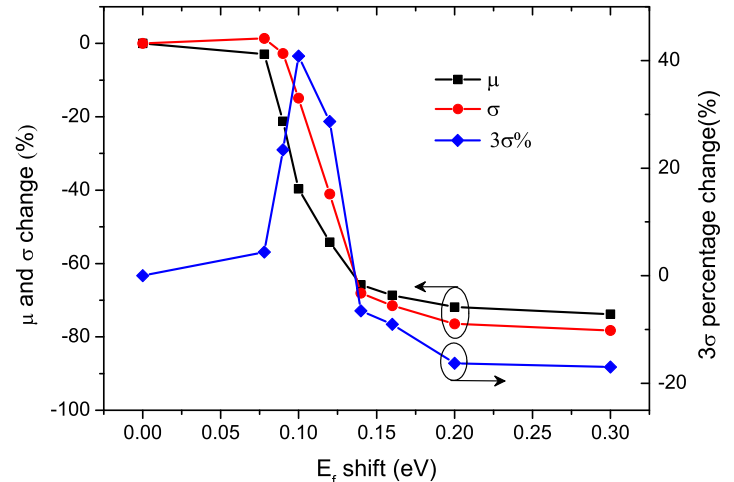

Fig. 9. Change of MWCNT resistivity and its variability from the all-sources simulation results with the $E_{f}$ shift for the MWCNT of $D_{\max }=6.5 \mathrm{~nm}$ (other parameters are the same to previous simulations).

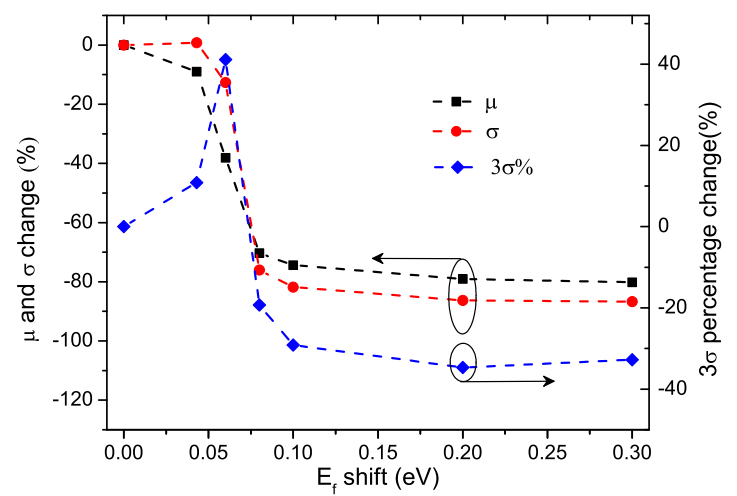

Fig. 10. Change of MWCNT resistivity and its variability from the all-sources simulation results with the $E_{f}$ shift for MWCNT of $D_{\max }=11 \mathrm{~nm}$ (MFP $\left.=1000 D_{C N T}\right)$.

of these doped CNTs are not measurable, direct comparisons of $\mu$ and $\sigma$ of MWCNT resistance between experiments and simulation (all-sources variation) are difficult and meaningless. Instead, we compare relative change (or doping efficiency) in MWCNT resistance $\mu$ and $\sigma$, and the $3 \sigma$ percentage variations with $E_{f}$ shift for MWCNTs of $D_{\max }=6.5 \mathrm{~nm}$. Fig. 9 shows the simulation results of MWCNT doping efficiency with $E_{f}$ shift. In Table II, we show doping efficiency of $\mu$ and $\sigma$, and $3 \sigma$ percentage variations before and after doping for experimental results (obtained this work or those from [20]). By comparing these simualtion reuslts and experimental results we can further predict the Fermi shift due to experimental doping, as presented in the table. For comparisons, the corresponding $\mu$, $\sigma$ and $3 \sigma$ relative percentage reductions from Fig. 9 are shown in Table II as well. The most significant deviation between the simulation results and experimental data is the relative change in $3 \sigma$ percentage variation at the $E_{f}$ shift of $0.122 \mathrm{eV}$. This can be attributed to the abrupt change in $3 \sigma$ percentage variation as shown in Fig. 9 and the assumed $E_{f}=0 \mathrm{eV}$ for pristine CNTs (before doping operation) which may be incorrect because pdoped CNTs have an $E_{f}$ downshift which can be expected during fabrication [4], [22].

Except for the $3 \sigma$ percentage deviation, an overall good match between experimental data and simulation results is observed, which strongly demonstrates the capability of the

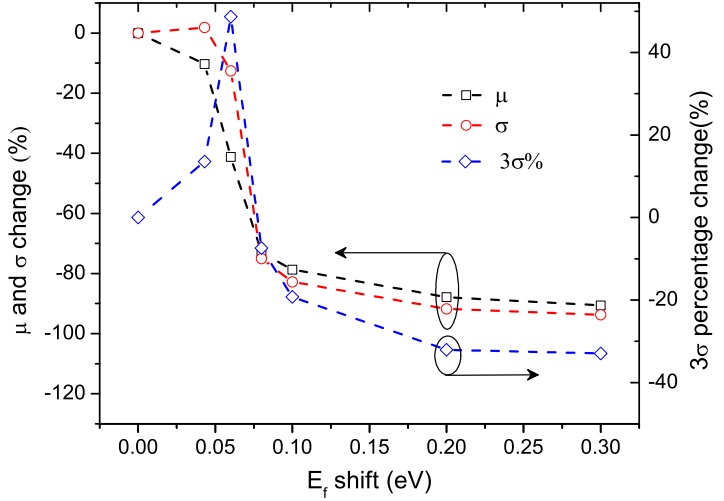

Fig. 11. Change of MWCNT resistivity and its variability from the all-sources simulation results with $E_{f}$ shift for MWCNT of $D_{\max }=11 \mathrm{~nm}($ MFP $=$ $\left.10 D_{C N T}\right)$.

MWCNT compact model to quantitatively predict relative change in MWCNT performance and the doping efficiency.

\section{Doping Efficiency}

1) Impact of MWCNT diameter: It is worth noting that the required level of the $E_{f}$ shift (or effective $E_{f}$ shift) to achieve a significant improvement of MWCNT resistance is dependent on the MWCNT dimensions though the overall change of doping efficiency with doping is similar. For example, by decreasing $D_{\max }$ from $11 \mathrm{~nm}$ to $6.5 \mathrm{~nm}$, the effective $E_{f}$ shift is increased from $\sim 0.1 \mathrm{eV}$ to $\sim 0.16 \mathrm{eV}$ but the overall change of doping efficiency with doping is similar, as shown in Fig. 9 and Fig. 10.

2) Impact of MFP: CNTs are of interest due to their ballistic transport and long mean free path (MFP). In this work, we assume that the default value of MFP in each shell of MWCNT is 1000 times the CNT shell diameter [24]. However, MFP is dependent on MWCNT fabrication process and thus may change from process to process. For instance, based on the scattering resistance of CVD-grown MWCNTs measured in [20] and in this work - it appears that MFP is shorter than $100 \mathrm{~nm}\left(\sim 10-20 \times D_{C N T}\right)$, which is also in agreement with other literature reports [28]. So, it is worth investigating the impact of MFP on doping efficiency by considering different MFPs. Simulation results of doping efficiency for MFP of 10 $\times D_{C N T}$ are presented in Fig. 11 and compared with the default case (MFP of $1000 \times D_{C N T}$ ) in Fig. 10. It shows that despite the 100 times difference in MFP, doping efficiency is similar. This is because doping increases $N_{C}$ of CNT shells and further improves conductance of MWCNT, which is impacted by MFP proportionally under different $N_{C}$ as shown in Equations (3) and (4). As a result, we deduce that the MFP does not impact the relative change of MWCNT resistance and performance improvements obtained from doping.

3) Impact of Pristine MWCNT chirality: As demonstrated in Section III-B, the improvement of MWCNT performance and variability with doping is mainly attributed to chirality variation reduction. Because the chirality variation results from the change of metallic and semiconducting CNT shell portions, it is reasonable to deduce that the doping efficiency can be 


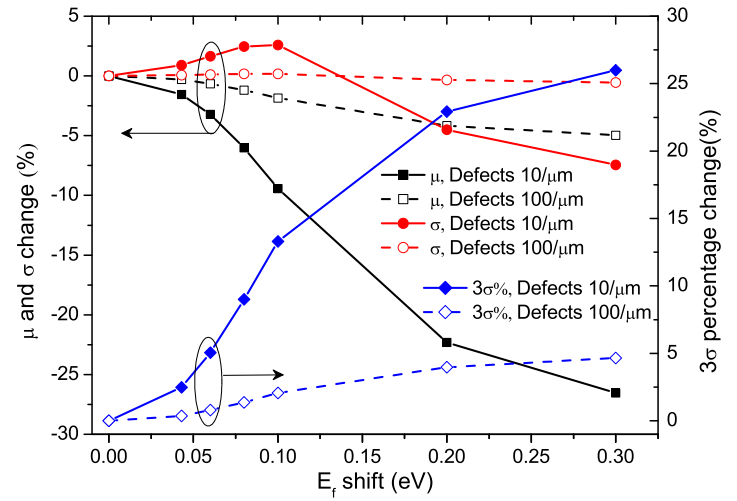

Fig. 12. Change of metallic MWCNT resistivity and its variability from the all-sources simulation results with the $E_{f}$ shift for defect density of $10 / \mu \mathrm{m}$ (the default) and $100 / \mu \mathrm{m}$.

degraded if the original MWCNT is more metallic, namely a larger portion of metallic CNT shells. For example, Fig. 12 shows the simulation results of entirely metallic MWCNT, namely metallic chirality 1 (other parameters are the same as the default one) with the $E_{f}$ shift. Compared with the default MWCNT (1/3 of metallic CNT shells) as shown in Fig. 10, the doping efficiency is reduced for the entirely metallic MWCNT. Instead of the abrupt reduction in $\mu$ and $\sigma$ of MWCNT resistance for the default MWCNT from the $E_{f}$ shift of 0.043 $\mathrm{eV}$ to $0.1 \mathrm{eV}$, a continuous and slow reduction is observed for the entirely metallic MWCNT up to $0.3 \mathrm{eV}$. Moreover, different from the default case where $3 \sigma$ percentage can finally be reduced by doping up to $0.1 \mathrm{eV}$, the $3 \sigma$ percentage is continuously increased with doping for the entire metallic MWCNT case.

4) Impact of Defect Density: We study the impact of defect density and in Fig. 12 we show the simulation results of doping efficiency for the entire metallic MWCNT. We observe that with the increase in defect density, doping effectiveness is significantly reduced for the entire metallic MWCNT. For example, the doping efficiency of $\mu$ and $\sigma$ of MWCNT resistance at the $0.3 \mathrm{eV} E_{f}$ shift is $26.5 \% / 7.3 \%$ and $5.0 \% / 0.7 \%$ for defect density of $10 / \mu \mathrm{m}$ and $100 / \mu \mathrm{m}$, respectively. The $3 \sigma$ percentage is increased relatively by $26.1 \%$ and $4.5 \%$, respectively. This is because the role of diameter and defect variations are relatively more important as defect density is increased for the case of entirely metallic MWCNT. However, it is found that for the default case of $1 / 3$ metallic chirality, the defect density impact on doping effectiveness is negligible (less than $2 \%$ and $4 \%$ difference are observed at the $E_{f}$ of $0.1 \mathrm{eV}$ and $0.3 \mathrm{eV}$, respectively). This is due to the dominant impact of chirality variation to the overall variation response with $E_{f}$ shift for the default case.

\section{E. MWCNT Shell to Contact Variations}

Here, we study the impact of doping on MWCNT shells connectivity to contact. In Fig. 13, we show the relative worst case (the outermost shells disconnected, as discussed in Part I of this paper) resistance degradation (similar to $\sigma$ ) versus a different number of disconnected shells for different $E_{f}$ shift

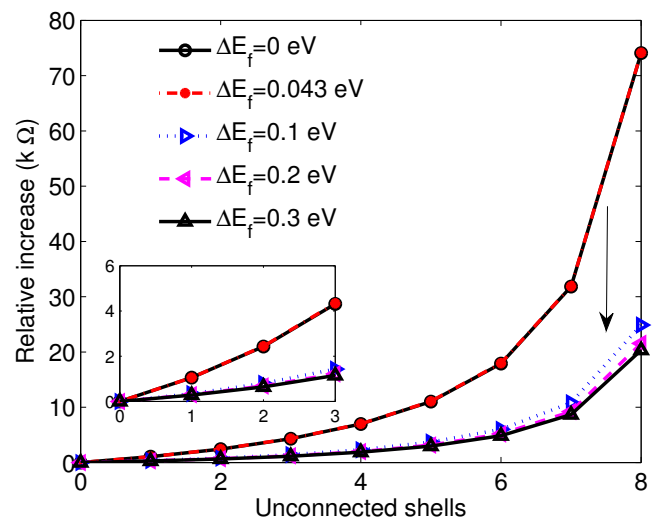

Fig. 13. Relative increase in the worst case MWCNT delay with several disconnected shells and $E_{f}$ shift levels compared to the case of all (nine) connected shells. The inset is the simulation result of unconnected shells from 0 to 3 after zooming in.

levels compared to all shells connected case. No significant improvements with doping are observed till $E_{f}=0.043 \mathrm{eV}$ and significant improvements are observed for $E_{f} \geq 0.1 \mathrm{eV}$. When normalizing the relative worst resistance degradation to the case with all shells connected resistance (similar to $\sigma / \mu$, namely relative variation), it is found that there is not an obvious impact from doping up to five disconnected shells (out of nine shells total) and increases slightly as more shells are disconnected. MWCNTs performances with disconnected shell have a similar response to doping and are not shown here. Hence, we deduce that doping improves MWCNT resistance and then performances, which allows mitigating the effect of disconnected shells without increasing relative variation significantly.

\section{Discussion AND CONCLUSION}

In this work, we show that by doping ( $E_{f}$ shift to $0.1 \mathrm{eV}$ ) on a MWCNT of $D_{\max }=11 \mathrm{~nm}$ significant improvements on performance and variability can be obtained. However, we also show that for MWCNT of smaller diameters, more doping or $E_{f}$ shift is required to achieve these improvements. Hence, as technology scales, the local interconnect sizes also decrease [29], which implies that MWCNT interconnects for advanced technology node applications may need higher doping levels to improve their performance and variability efficiently. Moreover, the $N_{C}$ of semiconducting CNT shells decrease with their diameter and technology scaling, hence, increasing the importance of applying doping to local interconnects with small geometries to improve MWCNT resistance and chirality variation. However, doping can do much. Other sources of variations such as diameter and defects will require further improvement on CNT process growth to exploit the potentials of MWCNTs for interconnect application.

In this work, we propose an enhanced compact model for MWCNT interconnects to take into account variability including diameter, defects, chirality and connectivity but also the impact of doping as a countermeasure to large resistance variability. Based on the analytical formulations, the change 
of MWCNT resistance and performance variation (including delay, power, and PDP) with $E_{f}$ shift can be calculated while including variations on diameter, defect densities, chirality, and shell-to-contact connectivity. We identify that chirality variation (both $\sigma$ and $3 \sigma$ percentage) can be significantly reduced to almost zero when the doping $E_{f}$ shift enables to degenerate all semiconducting shells to equivalent metallic like shell conductivity. Also, the diameter and defect variations $\sigma$ can be reduced up to $\sim 80 \%$ while the $3 \sigma$ percentage is slightly increased. The efficiency of doping is compared with experimental data, and an overall good match is obtained, which demonstrates the validity of computing doping efficiency and simulation methodology. We observe that MFP has a minor effect on doping efficiency. However, doping efficiency can be significantly reduced and degraded if the original portion of metallic CNT shells increases. Overall, we have established the analytical methods and simulation framework to study charge based doping impact on MWCNT interconnects performance and variability.

\section{ACKNOWLEDGMENT}

This project has received funding from the European Unions Horizon 2020 research and innovation program under grant agreement No 688612. (http://www.connect-h2020.eu/).

\section{REFERENCES}

[1] A. Todri-Sanial, J. Dijon, and A. Maffucci, Carbon Nanotubes for Interconnects. Springer, 2017 ISBN: 978-3-319-29744-6. doi: 10.1007/9783-319-29746-0

[2] K. Liew, C. Wong, X. He, and M. Tan, "Thermal stability of single and multi-walled carbon nanotubes," Physical Review B, vol. 71, no. 7, p. 075424, 2005. doi: 10.1103/physrevb.71.075424

[3] B. Wei, R. Vajtai, and P. Ajayan, "Reliability and current carrying capacity of carbon nanotubes," Applied Physics Letters, vol. 79, no. 8, pp. 1172-1174, 2001. doi: 10.1063/1.1396632

[4] W. Zhou, J. Vavro, N. M. Nemes, J. E. Fischer, F. Borondics, K. Kamaras, and D. Tanner, "Charge transfer and fermi level shift in p-doped single-walled carbon nanotubes," Physical Review B, vol. 71, no. 20, p. 205423, 2005. doi: 10.1103/physrevb.71.205423

[5] J. Liang, L. Zhang, N. Azemard-Crestani, P. Nouet, and A. TodriSanial, "Physical description and analysis of doped carbon nanotube interconnects," IEEE International Workshop on Power and Timing Modeling, Optimization and Simulations (PATMOS), pp. 250-255, 2016. doi: $10.1109 /$ patmos.2016.7833695

[6] M. Monthioux, Carbon meta-nanotubes: Synthesis, properties and applications. John Wiley \& Sons, 2011. doi: 10.1002/9781119954743

[7] L. Yu, C. Shearer, and J. Shapter, "Recent development of carbon nanotube transparent conductive films," Chemical reviews, vol. 116, no. 22, pp. 13 413-13 453, 2016. doi: 10.1021/acs.chemrev.6b00179

[8] S. L. Hellstrom, M. Vosgueritchian, R. M. Stoltenberg, I. Irfan, M. Hammock, Y. B. Wang, C. Jia, X. Guo, Y. Gao, and Z. Bao, "Strong and stable doping of carbon nanotubes and graphene by moo $\mathrm{x}$ for transparent electrodes," Nano letters, vol. 12, no. 7, pp. 3574-3580, 2012. doi: 10.1021/nl301207e

[9] B. Chandra, A. Afzali, N. Khare, M. M. El-Ashry, and G. S. Tulevski, "Stable charge-transfer doping of transparent single-walled carbon nanotube films," Chemistry of Materials, vol. 22, no. 18, pp. 5179-5183, 2010. doi: $10.1021 / \mathrm{cm} 101085 \mathrm{p}$

[10] J. E. Fischer, "Chemical doping of single-wall carbon nanotubes," Accounts of chemical research, vol. 35, no. 12, pp. 1079-1086, 2002. doi: 10.1021/ar0101638

[11] L. Kavan, P. Rapta, L. Dunsch, M. J. Bronikowski, P. Willis, and R. E. Smalley, "Electrochemical tuning of electronic structure of single-walled carbon nanotubes: in-situ raman and vis-nir study," The Journal of Physical Chemistry B, vol. 105, no. 44, pp. 10764-10771, 2001. doi: 10.1021/jp011709a
[12] J. Kong, N. R. Franklin, C. Zhou, M. G. Chapline, S. Peng, K. Cho, and H. Dai, "Nanotube molecular wires as chemical sensors," science, vol. 287, no. 5453, pp. 622-625, 2000. doi: 10.1126/science.287.5453.622

[13] V. Skakalova, A. Kaiser, U. Dettlaff-Weglikowska, K. Hrncarikova, and S. Roth, "Effect of chemical treatment on electrical conductivity, infrared absorption, and raman spectra of single-walled carbon nanotubes," The Journal of Physical Chemistry B, vol. 109, no. 15, pp. 7174-7181, 2005. doi: $10.1021 / \mathrm{jp} 044741$ o

[14] B. B. Parekh, G. Fanchini, G. Eda, and M. Chhowalla, "Improved conductivity of transparent single-wall carbon nanotube thin films via stable postdeposition functionalization," Applied Physics Letters, vol. 90, no. 12, p. 121913, 2007. doi: 10.1063/1.2715027

[15] Y. Zhao, J. Wei, R. Vajtai, P. M. Ajayan, and E. V. Barrera, "Iodine doped carbon nanotube cables exceeding specific electrical conductivity of metals," Scientific reports, vol. 1, p. 83, 2011. doi: 10.1038/srep00083

[16] S. Esconjauregui, L. DArsie, Y. Guo, J. Yang, H. Sugime, S. Caneva, C. Cepek, and J. Robertson, "Efficient transfer doping of carbon nanotube forests by moo3," ACS nano, vol. 9, no. 10, pp. 10422-10430, 2015. doi: 10.1021/acsnano.5b04644

[17] J. Dijon, R. Ramos, A. Fournier, H. Le Poche, H. Fournier, H. Okuno, and J. Simonato, "Record resistivity of in-situ grown horizontal carbon nanotube interconnect," in Technical proceedings of the 2014 NSTI nanotechnology conference and expo, NSTI-Nanotech, vol. 3, 2014, pp. 17-20. [Online]. Available: https://www.nsti.org/procs/Nanotech2014v3/ $1 / \mathrm{T} 2.154$

[18] B. Padya, D. Kalita, P. Jain, G. Padmanabham, M. Ravi, and K. Bhat, "Self-organized growth of bamboo-like carbon nanotube arrays for field emission properties," Applied Nanoscience, vol. 2, no. 3, pp. 253-259, 2012. doi: 10.1007/s13204-012-0102-z

[19] A. L. P. L. P. Petit, S. Roche, and J. Salvetat, Understanding Carbon Nanotubes, from Basics to Application. Lect. Notes Phys., édition Springer, 2006. doi: 10.1007/b10971390

[20] J. Liang, R. Ramos, J. Dijon, H. Okuno, D. Kalita et al., "A physicsbased investigation of pt-salt doped carbon nanotubes for local interconnects," in Electron Devices Meeting (IEDM), 2017 IEEE International. IEEE, 2017, pp. 35-5. doi: 10.1109/iedm.2017.8268502

[21] H. H. B. Sørensen, P. C. Hansen, D. E. Petersen, S. Skelboe, and K. Stokbro, "Efficient wave-function matching approach for quantum transport calculations," Physical Review B, vol. 79, no. 20, p. 205322, 2009. doi: 10.1103/physrevb.79.205322

[22] X. Ma, L. Adamska, H. Yamaguchi, S. E. Yalcin, S. Tretiak, S. K. Doorn, and H. Htoon, "Electronic structure and chemical nature of oxygen dopant states in carbon nanotubes," ACS nano, vol. 8, no. 10, pp. 10782-10789, 2014. doi: 10.1021/nn504553y

[23] S. Datta, Quantum transport: atom to transistor. Cambridge university press, 2005. doi: 10.1017/cbo9781139164313

[24] H. Li, W.-Y. Yin, K. Banerjee, and J.-F. Mao, "Circuit modeling and performance analysis of multi-walled carbon nanotube interconnects," IEEE Transactions on electron devices, vol. 55, no. 6, pp. 1328-1337, 2008. doi: 10.1109/ted.2008.922855

[25] C.-S. Lee, E. Pop, A. D. Franklin, W. Haensch, and H.-S. Wong, "A compact virtual-source model for carbon nanotube fets in the sub-10-nm regimepart i: Intrinsic elements," IEEE transactions on electron devices, vol. 62, no. 9, pp. 3061-3069, 2015. doi: 10.1109/ted.2015.2457453

[26] A. Nieuwoudt and Y. Massoud, "On the impact of process variations for carbon nanotube bundles for vlsi interconnect," IEEE Transactions on Electron Devices, vol. 54, no. 3, pp. 446-455, 2007. doi: 10.1109/ted.2006.890364

[27] F. Pukelsheim, "The three sigma rule," The American Statistician, vol. 48, no. 2, pp. 88-91, 1994. doi: 10.1080/00031305.1994.10476030

[28] A. A. Vyas, C. Zhou, and C. Y. Yang, "On-chip interconnect conductor materials for end-of-roadmap technology nodes," IEEE Transactions on Nanotechnology, 2016. doi: 10.1109/tnano.2016.2635583

[29] L. Wilson, "International technology roadmap for semiconductors (itrs)," Semiconductor Industry Association, 2013. [Online]. Available: http:// www.itrs2.net/2013-itrs.html 